\title{
Charge Asymmetry in Top Pairs at ATLAS
}

\section{Mario P. Giordani*}

On behalf of the ATLAS Collaboration

Università degli Studi di Udine \& INFN Sezione di Trieste

E-mail: mario.giordani@uniud.it

In $7 \mathrm{TeV}$ proton-proton collisions at the LHC, pairs of top and anti-top quarks are expected to be mostly produced through gluon fusion, in contrast to production at the Tevatron, where quark annihilation dominates. The ATLAS experiment has now recorded a large number of top quark pairs, allowing this domain to be explored in detail. We present measurements of top quark charge asymmetry which constitute important tests of QCD and are sensitive to new physics. Measurements are presented in both the single lepton and dilepton channels. Also, the leptonbased charge asymmetry in top quark pair events has been measured and will be presented.

36th International Conference on High Energy Physics,

July 4-11, 2012

Melbourne, Australia

* Speaker. 


\section{Introduction}

Since its discovery dating back to 1995 , the top quark has played a primary role in high-energy physics; due to its large mass, in the close range of the electroweak scale, it is considered to be an important probe for both the Standard Model (SM) and other theories incorporating new physics phenomena. The production of pairs of top and anti-top quarks is characterized by a significant cross-section at the LHC, which makes this process - and the top quark itself - a key ingredient to precision tests of the properties predicted by Quantum Chromodynamics (QCD) at high energies.

\section{The Origin of Charge Asymmetry}

Although at leading order (LO) the production of q $\bar{q}$ pairs is predicted to be symmetric under charge conjugation, next-to-leading order (NLO) corrections are expected to introduce asymmetries in the differential quark and anti-quark distributions: as a result, top quarks (anti-quarks) are expected to be emitted preferentially in the direction of the incoming quark (anti-quark). Interference between the $\mathrm{q} \overline{\mathrm{q}} \rightarrow \mathrm{t} \overline{\mathrm{t}}$ Born-level and box diagrams leads to an effect which is partially compensated by the inclusion of initial and final state radiation contributions ( $\mathrm{q} \overline{\mathrm{q}} \rightarrow \mathrm{t} \overline{\mathrm{tg}}$ ). Smaller corrections are due to the qg $\rightarrow \mathrm{ttq}$ subprocess, while no effect is expected from gluon-fusion. It is then understood that the enhancement of concurrent gluon-initiated mechanisms eventually dilutes the magnitude of the observed charge asymmetry.

\subsection{Definition of a Suitable Charge Asymmetry Observable}

At the Tevatron, where $t \bar{t}$ were yielded predominantly by $q \bar{q}$ annihilation in $p \bar{p}$ collisions, top quarks (anti-quarks) were produced preferentially in the proton (anti-proton) beam direction; their charge asymmetry could therefore be measured as a forward-backward asymmetry in the $t \bar{t}$ rest frame:

$$
\mathscr{A}_{f b}=\frac{N\left(\cos \theta^{*}>0\right)-N\left(\cos \theta^{*}<0\right)}{N\left(\cos \theta^{*}>0\right)+N\left(\cos \theta^{*}<0\right)}=\frac{N\left(y_{\mathrm{t}}>0\right)-N\left(y_{\mathrm{t}}<0\right)}{N\left(y_{\mathrm{t}}>0\right)+N\left(y_{\mathrm{t}}<0\right)}=\frac{N(\Delta y>0)-N(\Delta y<0)}{N(\Delta y>0)+N(\Delta y<0)},
$$

where $N$ indicates the number of events, $\theta^{*}$ is the angle of emission of the top quark with respect to the incoming proton and $y_{\mathrm{t}}$ is the top quark rapidity - with both $\theta^{*}$ and $y_{\mathrm{t}}$ being measured in the $\mathrm{t} \overline{\mathrm{t}}$ frame. In the last equality, resulting from properties of rapidity, the Lorentz-invariant $\Delta y=y_{\mathrm{t}}-y_{\mathrm{t}}$ can be used to compute $\mathscr{A}_{f b}$ from top quark and anti-quark rapidities measured in the more convenient laboratory frame.

At the LHC, where the incoming beams are identical and the t⿱t production is dominated by gluon-fusion, the ambiguity in the definition of colliding quark (as opposed to anti-quark) makes $\mathscr{A}_{f b}$ no longer an observable of interest. However, since top quarks (anti-quarks) are preferentially emitted in the direction of the incoming quark (anti-quark), and given that colliding quarks are typically associated to the more energetic valence partons as opposed to colliding anti-quarks (more likely originating from the sea and therefore carrying a smaller momentum), top quarks are expected to be more boosted than top anti-quarks, i.e. $\Delta|y| \equiv\left|y_{\mathrm{t}}\right|-\left|y_{\hat{\mathrm{t}}}\right|$ is expected to be positive.

As a consequence, a new observable can be built to study the production asymmetry:

$$
\mathscr{A}_{C}=\frac{N(\Delta|y|>0)-N(\Delta|y|<0)}{N(\Delta|y|>0)+N(\Delta|y|<0)} .
$$


The charge asymmetry definition given in eq. 2.2 can be used in all cases in which a complete reconstruction of the $t \bar{t}$ system can be achieved or at least inferred (such as in the semi-leptonic decay channel); in dilepton final states, where the two neutrinos under-constrain the kinematics, a lepton-based charge asymmetry can be measured by considering the pseudorapidity of the outgoing leptons instead of the rapidity of the top quarks or anti-quarks:

$$
\mathscr{A}_{C}^{\ell \ell}=\frac{N(\Delta|\eta|>0)-N(\Delta|\eta|<0)}{N(\Delta|\eta|>0)+N(\Delta|\eta|<0)}
$$

where $\Delta|\eta| \equiv\left|\eta_{\ell^{+}}\right|-\left|\eta_{\ell^{-}}\right|$. Despite of relying on the precise determination of the lepton directions, $\mathscr{A}_{C}^{\ell \ell}$ targets a weaker effect: the charge asymmetry is diluted by the kinematics of top quark and anti-quark decays. Furthermore, $\mathscr{A}_{C}^{\ell \ell}$ is expected to be sensitive to top polarization effects.

\section{Analyses Outline}

The measurement of the charge asymmetry in $t \bar{t}$ events has been performed on data collected by the ATLAS detector [1] during 2011 at $\sqrt{s}=7 \mathrm{TeV}$. Events selected by an inclusive-lepton trigger have been categorized in a $1.04 \mathrm{fb}^{-1}$ single-lepton sample and a $4.71 \mathrm{fb}^{-1}$ dilepton sample and processed by two different analyses targeting either semileptonic or fully leptonic $t \bar{t}$ decays $[2,3]$.

The two procedures share the common goal of reconstructing the $t \bar{t}$ system, a necessary step in order to measure the rapidities of top quarks and anti-quarks, and rely on the same definition of physics objects, i.e. leptons, jets and missing transverse energy $\left(E_{\mathrm{T}}^{m i s}\right)$. Electrons and muons are required to be energetic ( $p_{\mathrm{T}}>25$ and $20 \mathrm{GeV}$ respectively), isolated and identified in the central part of the detector $\left(\left|\eta_{\mathrm{e}}\right|<2.47\right.$ and $\left.\left|\eta_{\mu}\right|<2.5\right)$. Jets are reconstructed by means of the anti- $k_{\mathrm{T}}$ algorithm with $R=0.4$ and $\left|\eta_{j e t}\right|<2.5$; their energy is calibrated according to standard ATLAS procedures and required to fulfil $E_{\mathrm{T}}>25 \mathrm{GeV}$. The computation of $E_{\mathrm{T}}^{\text {miss }}$ relies on the energy depositions detected in the calorimeter calibrated to the electromagnetic energy scale and corrected to the energy scale of the associated physics objects; corrections due to the presence of muons are also applied.

Backgrounds like $\mathrm{W}+$ jets and QCD multijet involving the contamination of fake leptons ${ }^{1}$ have been derived by means of data-driven methods. All remaining backgrounds, which lead to the production of genuine prompt leptons (such as single top, $\mathrm{Z}+$ jets and diboson production), have been estimated by means of suitable Monte Carlo simulations.

\subsection{Single-lepton Channel}

Signal events are characterized by exactly one energetic lepton, $E_{\mathrm{T}}^{\text {miss }}$ due to the neutrino escaping detection and four jets, two of which originating from $b$ quark decays. Contamination due to QCD multijet production is reduced by a combined requirement on $E_{\mathrm{T}}^{\text {miss }}$ and on $m_{\mathrm{T}}(\mathrm{W})$, the transverse mass of the $\mathrm{W}$ boson: $E_{\mathrm{T}}^{\text {miss }}>35 \mathrm{GeV}, m_{\mathrm{T}}(\mathrm{W})>25 \mathrm{GeV}$ and $E_{\mathrm{T}}^{\text {miss }}>20 \mathrm{GeV}$, $E_{\mathrm{T}}^{m i s s}+m_{\mathrm{T}}(\mathrm{W})>60 \mathrm{GeV}$ are applied to the electron and muon channels respectively. At least one jet is required to be identified as originated from a $b$ quark; jet b-tagging involves two different

\footnotetext{
${ }^{1}$ Fake leptons are generally originated by either jets misidentified as leptons or by non-prompt real leptons.
} 

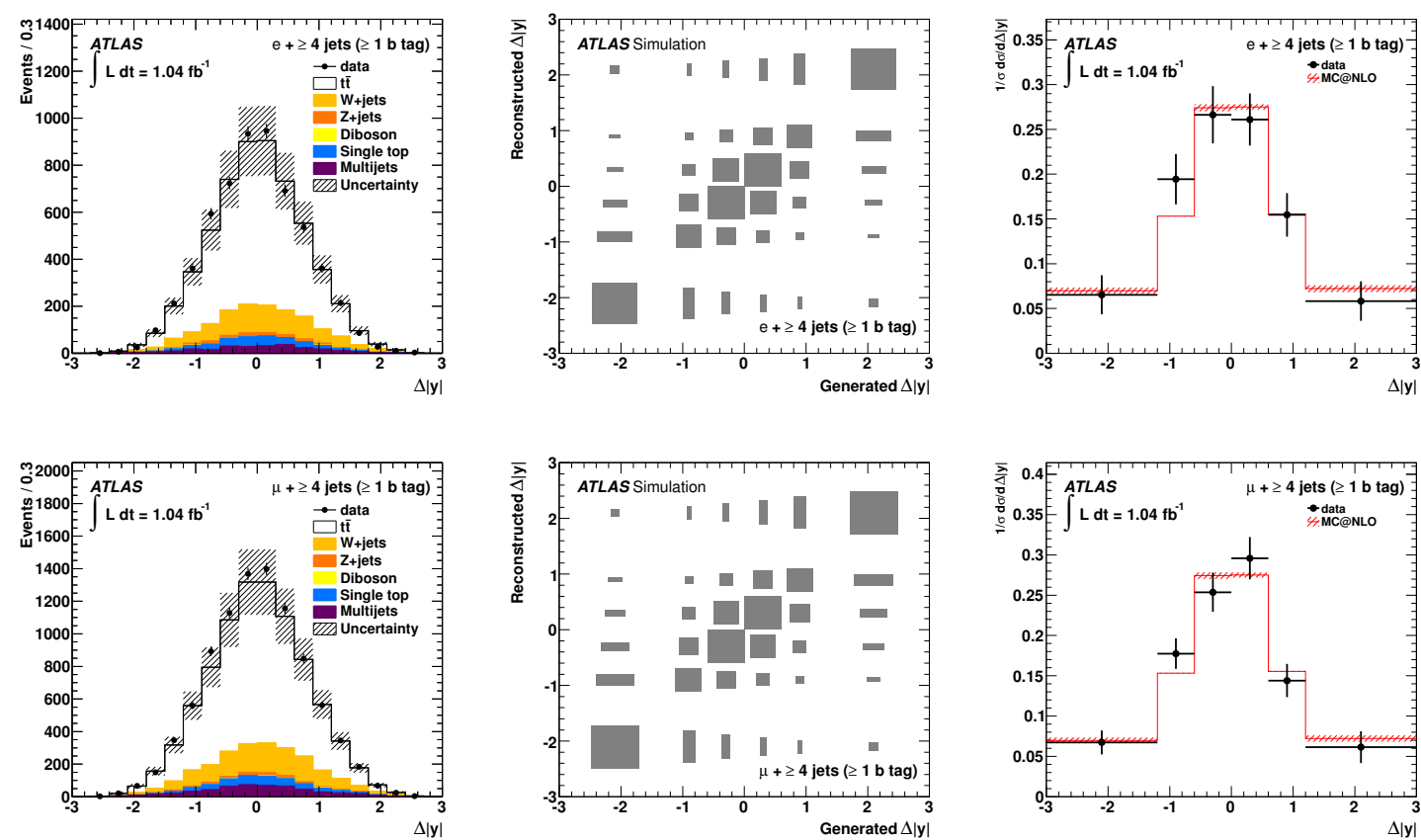

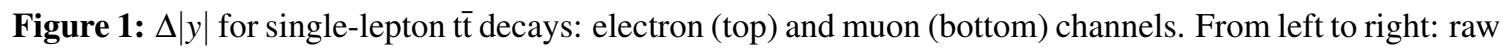
$\Delta|y|$ distributions, unfolding response matrices and unfolded background-subtracted $\Delta|y|$ spectra compared with Monte Carlo parton-level SM predictions [2].

algorithms, one based on the explicit secondary vertex reconstruction and the other one relying on a track-based transverse and longitudinal impact parameter significance calculation.

The reconstruction of the $t \bar{t}$ system exploits a kinematic fit that assesses the compatibility of the observed events with the topology of $t \bar{t}$ decays on the basis of a likelihood approach; the combination corresponding to the maximum likelihood ${ }^{2}$ is retained and used in the $\mathscr{A}_{C}$ measurement.

\subsection{Dilepton Channel}

The signature characterizing signal events includes two oppositly-charged energetic leptons, $E_{\mathrm{T}}^{\text {miss }}$ arising from the two neutrinos and two jets originating from heavy-flavour decay. Three channels are identified according to their lepton flavour content: $\mathrm{e}^{ \pm} \mathrm{e}^{\mp}, \mathrm{e}^{ \pm} \mu^{\mp}$ and $\mu^{ \pm} \mu^{\mp}$. In order to remove $\mathrm{Z}+$ jets contamination, the $\mathrm{Z}$-veto requirements $\left|m_{\ell^{+} \ell^{-}}-m_{\mathrm{Z}}\right|>10 \mathrm{GeV}$ and $E_{\mathrm{T}}^{\text {miss }}>60 \mathrm{GeV}$ have been applied to $\mathrm{e}^{ \pm} \mathrm{e}^{\mp}$ and $\mu^{ \pm} \mu^{\mp}$ events; $\mathrm{e}^{ \pm} \mu^{\mp}$ events, on the other hand, are required to fulfill $\Sigma_{\ell, j e t}\left(E_{\mathrm{T}}^{\ell}+E_{\mathrm{T}}^{\text {jet }}\right)>130 \mathrm{GeV}$.

Having two neutrinos in the final state, the kinematics of t $\bar{t}$ decays is under-constrained; due to finite energy-momentum resolution, several solutions are admissible. In every event, each solution has been weighted according to a likelihood estimator derived from matrix elements for the process $\mathrm{gg} \rightarrow \mathrm{t} \overline{\mathrm{t}} \rightarrow \ell^{+} v_{\ell} \mathrm{b} \ell^{-} \bar{v}_{\ell} \overline{\mathrm{b}}$ and further considered in charge asymmetry measurements.

\footnotetext{
${ }^{2}$ This method identifies the correct topology in the $74 \%$ of the cases.
} 


\begin{tabular}{c|cc} 
& Data & MC@ NLO \\
\hline $\mathscr{A}_{C}(\mathrm{e}+$ jets $)$ & $-0.047 \pm 0.045($ stat $) \pm 0.028($ syst $)$ & \\
$\mathscr{A}_{C}(\mu+$ jets $)$ & $-0.002 \pm 0.036($ stat $) \pm 0.023($ syst $)$ & $0.006 \pm 0.002$ \\
$\mathscr{A}_{C}(\ell+$ jets $)$ & $-0.018 \pm 0.028($ stat $) \pm 0.023($ syst $)$ & $0.006 \pm 0.002$ \\
$m_{\mathrm{tt}}<450 \mathrm{GeV}$ & $-0.053 \pm 0.070($ stat $) \pm 0.054($ syst $)$ & $0.005 \pm 0.002$ \\
$m_{\mathrm{tt}}>450 \mathrm{GeV}$ & $-0.008 \pm 0.035($ stat $) \pm 0.032($ syst $)$ & $0.007 \pm 0.002$ \\
\hline $\mathscr{A}_{C}\left(\mathrm{e}^{+} \mathrm{e}^{-}+\right.$jets $)$ & $0.079 \pm 0.087($ stat $) \pm 0.028($ syst $)$ & \\
$\mathscr{A}_{C}\left(\mathrm{e}^{ \pm} \mu^{\mp}+\right.$ jets $)$ & $0.078 \pm 0.029($ stat $) \pm 0.017($ syst $)$ & $0.006 \pm 0.002$ \\
$\mathscr{A}_{C}\left(\mu^{+} \mu^{-}+\right.$jets $)$ & $0.000 \pm 0.046($ stat $) \pm 0.021($ syst $)$ & \\
$\mathscr{A}_{C}(\ell \ell+$ jets $)$ & $0.057 \pm 0.024($ stat $) \pm 0.015($ syst $)$ & $0.006 \pm 0.002$ \\
\hline $\mathscr{A}_{C}(\ell+j e t s, \ell \ell+$ jets combined $)$ & $0.029 \pm 0.018($ stat $) \pm 0.014($ syst $)$ & $0.006 \pm 0.002$ \\
\hline $\mathscr{A}_{C}^{\mathrm{ee}}$ & $0.091 \pm 0.041($ stat $) \pm 0.029($ syst $)$ & \\
$\mathscr{A}_{C}^{\mathrm{e} \mu}$ & $0.018 \pm 0.014($ stat $) \pm 0.009($ syst $)$ & $0.004 \pm 0.001$ \\
$\mathscr{A}_{C}^{\mu \mu}$ & $0.026 \pm 0.023($ stat $) \pm 0.009($ syst $)$ & \\
$\mathscr{A}_{C}^{\ell \ell}$ & $0.023 \pm 0.012($ stat $) \pm 0.008($ syst $)$ & $0.004 \pm 0.001$
\end{tabular}

Table 1: Measurements of charge asymmetry in t⿱t decays compared with MC@NLO Monte Carlo predictions $[2,3]$.

\section{Results}

The observed distributions for the rapidity difference $\Delta|y|$ between top quarks and anti-quarks is expected to be distorted by detector inhomogeneities, finite acceptance and selection biases. It is therefore important to disentangle these effects from the underlying physics processes in order to provide a measurement that can be compared with theory predictions.

In the single-lepton analysis, an unfolding procedure has been developed; detector and acceptance-induced effects are folded in a response matrix relating true to observed quantities:

$$
O_{i}=\Sigma_{j} R_{i j} T_{j}
$$

where $R_{i j}$ describes the probability of observing in bin $i$ what is expected in bin $j$. True distributions $\left(T_{j}\right)$ can be obtained from observed distributions after background subtraction $\left(O_{i}\right)$ via matrix inversion. Response matrices have been derived by means of MC@NLO Monte Carlo simulations, while the matrix inversion has been performed by an iterative procedure involving Bayes' theorem. Raw $\Delta|y|$ distributions together with response matrices and unfolded spectra are shown in figure 1.

The approach followed by the dilepton analysis is based on a calibration technique. The correction has been estimated on the basis of tt samples generated with the MC@NLO Monte Carlo, in which events have been reweighted in order to inject a charge asymmetry of known magnitude; the charge asymmetry has then been measured from these manipulated samples and a reconstructed vs. true asymmetry calibration curve has been extracted. This method has been eventually used on $\mathrm{t} \overline{\mathrm{t}}$ data events after background subtraction.

A summary of the results achieved in the measurements of charge asymmetry in $t \bar{t}$ events performed by the ATLAS Collaboration is reported in table 1; the comparison between data and Monte Carlo shows no clear evidence of significant deviations.

The systematic uncertainties affecting the charge asymmetry measurements can be grouped in two classes: detector response and modelling of signal and background. The first category is dom- 

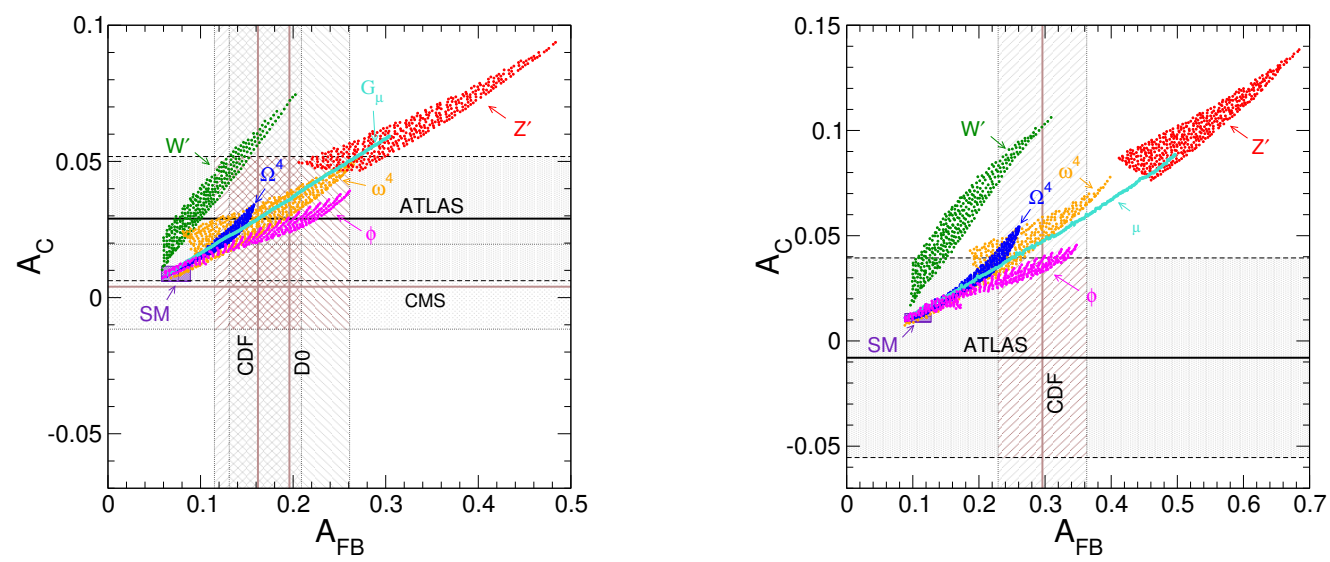

Figure 2: Experimental results [2, 3, 4, 5] compared to theoretical predictions provided by the SM and other models incorporating potential new physics contributions [6,7]. Left: inclusive $m_{\mathrm{tt}}$ data with ATLAS $\ell+$ jets and $\ell \ell+$ jets combination. Right: $m_{\mathrm{ti}} \geqslant 450 \mathrm{GeV}$ data with ATLAS $\ell+$ jets.

inated by the determination of the jet energy scale; the description of the fragmentation phase, the amount of initial and final state radiation, the simulation of the t⿱t process and the normalization of the $\mathrm{W}+$ jets background instead determine the most relevant contributions to the second category.

\section{Conclusion}

The charge asymmetry in t⿱t events has been measured by the ATLAS Collaboration in singlelepton and dilepton samples of data collected during 2011 at $\sqrt{s}=7 \mathrm{TeV}$ and corresponding to an integrated luminosity of $1.04 \mathrm{fb}^{-1}$ and $4.71 \mathrm{fb}^{-1}$ respectively. Results are in agreement with the SM $\mathscr{A}_{C}$ and $\mathscr{A}_{C}^{\ell \ell}$ predictions. These measurements have been used to constrain models that introduce new physics phenomena, some of which were inspired by an excess initially observed in $\mathscr{A}_{f b}$ measurements at the Tevatron. Figure 2 shows the current status of charge asymmetry measurements at hadron colliders compared with a plethora of predictions suggested by different extensions of the SM [6,7]. As it can be seen, the experimental results seem to consistently disfavour models predicting the existence of right-handed flavour-changing $Z^{\prime}$ bosons capable of

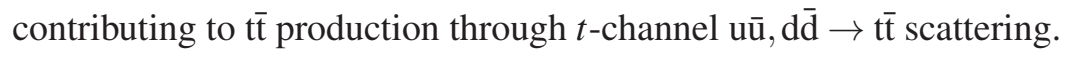

\section{References}

[1] ATLAS Collaboration, JINST 3 S08003 (2008)

[2] ATLAS Collaboration, Eur. Phys. J. C 72, 2039 (2012) and references quoted therein

[3] ATLAS Collaboration, ATLAS Note ATLAS-CONF-2012-057 (2012)

[4] CDF Collaboration, CDF Note CDF/ANAL/TOP/PUBLIC/10807 and references quoted therein

[5] CMS Collaboration, CMS Note CMS PAS TOP-11-030 and references quoted therein

[6] J. A. Aguilar-Saavedra and M. Perez-Victoria, Phys. Rev. D 83, 115013 (2011)

[7] J. A. Aguilar-Saavedra and M. Perez-Victoria, JHEP 1109, 097 (2011) 